\title{
Light waves scattering from an anisotropic semi-soft boundary medium with spectral dependence
}

\author{
ZhiRu HUANG, QIHANG DAI, XIAOLING JI, TAO WANG* \\ Department of Physics, Sichuan Normal University, Chengdu 610068, China \\ ${ }^{*}$ Corresponding author: towerwang@126.com
}

\begin{abstract}
The far-zone behavior of polychromatic light waves on scattering from an anisotrophic semi-soft boundary medium with spectral dependence was considered, and the spectral density and the spectral degree of coherence of the far-zone scattered field were investigated. It is shown that the distributions of the spectral density and the spectral degree of coherence of scattered field are closely related with the rms width, the center wavelength, and the maximum value of the center wavelength of the scattering potential of the scattering medium.
\end{abstract}

Keywords: light scattering, spectral dependent medium, spectral density, spectral degree of coherence.

\section{Introduction}

Light waves scattering, which researches the relation between the behaviors of the scattered field and the characteristics of the scattering media, is a considerable important topic due to its potential applications in areas such as target detection, remote sensing, medical diagnosis and so on. During the past few decades, numerous papers on light wave scattering have been published, which focus on the influence of characteristics of various media on the behaviors of the scattered field [1-10]. Among all of the properties of the scattered field, the spectral density and the spectral degree of coherence, which can be used to determine the structural information of the unknown object [11- $\underline{16}$ ], are always two important factors which attracted much attentions. For instance, the spectral density and the spectral degree of coherence of light wave on scattering from various media, including random medium [17-20], deterministic medium [21], quasi-homogeneous medium [ $\underline{5}-\underline{9}]$, collection of particles [ 222-25], were discussed respectively. And it is shown that the spectral density and spectral degree of coherence in the scattered field are closely related to the characteristics of the scatterer (for a review on these researches, please see [르] $)$.

On the other hand, to simplify the discussion, almost all the scattering media are assumed to be independent of the wavelength or frequency of the incident wave field. However, in fact, the electric vector of light may give rise to polarization of the scattering medium, and the polarization will bring about a change of the dielectric sus- 
ceptibility and the refractive index of the scattering medium $[\underline{18}, \underline{27}]$. Therefore, some previous papers have been published to discuss the issue of the properties of the far-zone scattered field that involve the effects of the wavelength of the incident field on the scattering media $[\underline{18}, \underline{28}]$. However, as far as we know, almost all the publications on this issue are based on isotropic medium model with spectral dependence. In this manuscript, we would like to extend the analysis from isotropic medium to the anisotropic one. First of all, the expressions of the spectral density and the spectral degree of coherence of light waves on scattering from anisotropic semi-soft boundary medium with spectral dependence are derived. After that, some numerical results are presented to show the influence of the characteristics of the scattering medium on the far-zone behaviors.

\section{Theory}

We now consider a polychromatic light wave, with a propagation direction specified by a unit vector $\mathbf{s}_{0}$, which is incident upon a random medium (as shown in Fig. 1). Its cross-spectral density function of incident field can be expressed as [르]

$$
W^{(i)}\left(\mathbf{r}_{1}^{\prime}, \mathbf{r}_{2}^{\prime} ; \omega\right)=S^{(i)}(\omega) \exp \left[i k \mathbf{s}_{0} \cdot\left(\mathbf{r}_{2}^{\prime}-\mathbf{r}_{1}^{\prime}\right)\right]
$$

where $S^{(i)}(\omega)$ represents the spectrum of incident wave, $\mathbf{r}_{1}^{\prime}$ and $\mathbf{r}_{2}^{\prime}$ are a pair of point vectors within the area of scattering medium, $k=\omega / c$ is the wave number with $c$ being the speed of light in vacuum. Now we assume that the spectrum of the incident field has a form of [29]

$$
S^{(i)}(\omega)=B \exp \left[\frac{\left(\omega-\omega_{0}\right)^{2}}{2 \Gamma_{0}}\right]
$$

where both parameters $B$ and $\Gamma_{0}$ are constant, and $\omega_{0}$ denotes the central frequency of the spectrum. And the cross-spectral density function of the scattered field can also be expressed as [29]

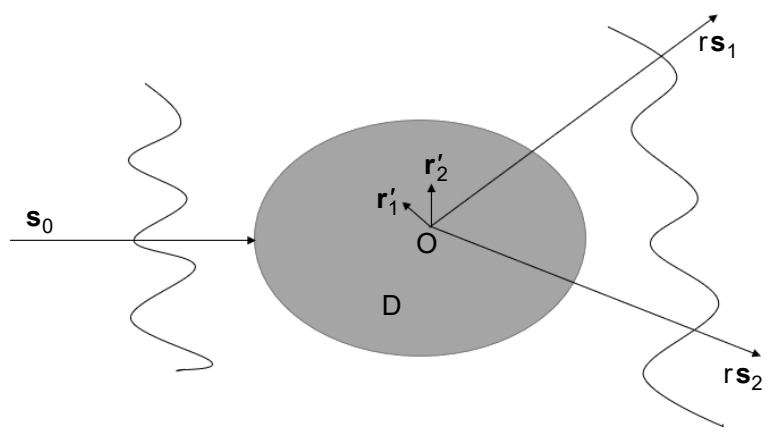

Fig. 1. Illustration of the notations. 


$$
\begin{aligned}
W^{(s)}\left(r \mathbf{s}_{1}, r \mathbf{s}_{2} ; \omega\right)=S^{(i)}(\omega) \iint_{D D} & \exp \left[i k \mathbf{s}_{0} \cdot\left(\mathbf{r}_{2}^{\prime}-\mathbf{r}_{1}^{\prime}\right)\right] C_{\mathrm{F}}\left(\mathbf{r}_{1}^{\prime}, \mathbf{r}_{2}^{\prime}, \omega\right) \\
& \times G^{*}\left(\left|r \mathbf{s}_{1}-\mathbf{r}_{1}^{\prime}\right|, \omega\right) G\left(\left|r \mathbf{s}_{2}-\mathbf{r}_{2}^{\prime}\right|, \omega\right) \mathrm{d}^{3} \mathbf{r}_{1}^{\prime} \mathrm{d}^{3} \mathbf{r}_{2}^{\prime}
\end{aligned}
$$

where $C_{\mathrm{F}}\left(\mathbf{r}_{1}^{\prime}, \mathbf{r}_{2}^{\prime}, \omega\right)$ is the correlation function of the scattering potential, and $G\left(\left|r \mathbf{s}-\mathbf{r}^{\prime}\right|, \omega\right)$ is the Green's function, which has an approximate form in the far field, i.e. [29],

$$
G\left(\left|r \mathbf{s}-\mathbf{r}^{\prime}\right|, \omega\right) \approx \frac{\exp (i k r)}{r} \exp \left(-i k \mathbf{s} \cdot \mathbf{r}^{\prime}\right)
$$

Upon substituting from Eq. (4) into Eq. (3), one can obtain the expression of the cross-spectral density function of the far-zone scattered field, which can be expressed as

$$
W^{(s)}\left(r \mathbf{s}_{1}, r \mathbf{s}_{2} ; \omega\right)=\frac{S^{(i)}(\omega)}{r^{2}} \tilde{C}_{\mathrm{F}}\left[-k\left(\mathbf{s}_{1}-\mathbf{s}_{0}\right), k\left(\mathbf{s}_{2}-\mathbf{s}_{0}\right), \omega\right]
$$

where

$$
\tilde{C}_{\mathrm{F}}\left[\mathbf{K}_{1}, \mathbf{K}_{2}, \omega\right]=\iint_{D D} C_{\mathrm{F}}\left(\mathbf{r}_{1}^{\prime}, \mathbf{r}_{2}^{\prime}, \omega\right) \exp \left[-i\left(\mathbf{K}_{1} \cdot \mathbf{r}_{1}^{\prime}+\mathbf{K}_{2} \cdot \mathbf{r}_{2}^{\prime}\right)\right] \mathrm{d}^{3} \mathbf{r}_{1}^{\prime} \mathrm{d}^{3} \mathbf{r}_{2}^{\prime}
$$

is the six-dimensional spatial Fourier transform of the correlation function $C_{\mathrm{F}}\left(\mathbf{r}_{1}^{\prime}, \mathbf{r}_{2}^{\prime}, \omega\right)$ of the scattering potential with $\mathbf{K}_{1}=-k\left(\mathbf{s}_{1}-\mathbf{s}_{0}\right)$ and $\mathbf{K}_{2}=k\left(\mathbf{s}_{2}-\mathbf{s}_{0}\right)$.

In some previous papers, the behaviors of light waves on scattering from an isotropic spectral-dependent medium were discussed [18, 28]. In this manuscript, we will generalize this discussion to a more general scattering model, i.e., an anisotropic semi-soft -boundary medium with spectra-dependence. Base on the spectral-codependent scattering model and the description of anisotropic medium $[\underline{28}, \underline{30}]$, one can present the correlation function of an anisotropic semi-soft-boundary medium with spectral dependence, with the form of

$$
\begin{aligned}
C_{\mathrm{F}}\left(\mathbf{r}_{1}^{\prime},\right. & \left.\mathbf{r}_{2}^{\prime}, \lambda\right)=\exp \left[-\frac{\left(x_{1}^{\prime}+x_{2}^{\prime}\right)^{2}}{8 \sigma_{x}^{2}(\lambda)}-\frac{\left(y_{1}^{\prime}+y_{2}^{\prime}\right)^{2}}{8 \sigma_{y}^{2}(\lambda)}-\frac{\left(z_{1}^{\prime}+z_{2}^{\prime}\right)^{2}}{8 \sigma_{z}^{2}(\lambda)}\right] \\
& \times \frac{1}{C_{0}} \sum_{m=1}^{M}\left(\begin{array}{c}
M \\
m
\end{array}\right) \frac{(-1)^{m-1}}{m^{3 / 2}} \exp \left[-\frac{\left(x_{1}^{\prime}-x_{2}^{\prime}\right)^{2}}{2 m \delta_{x}^{2}(\lambda)}-\frac{\left(y_{1}^{\prime}-y_{2}^{\prime}\right)^{2}}{2 m \delta_{y}^{2}(\lambda)}-\frac{\left(z_{1}^{\prime}-z_{2}^{\prime}\right)^{2}}{2 m \delta_{z}^{2}(\lambda)}\right]
\end{aligned}
$$

where

$$
C_{0}=\sum_{m=1}^{M} \frac{(-1)^{m-1}}{m^{3 / 2}}\left(\begin{array}{l}
M \\
m
\end{array}\right)
$$


is a normalization factor and

$$
\left(\begin{array}{l}
M \\
m
\end{array}\right)=\frac{M !}{m !(M-m) !}
$$

is the binomial coefficient with $M$ being a critical parameter that governs the boundary property of the scattering medium. In addition,

$$
\begin{aligned}
& \sigma_{i}(\lambda)=\sigma_{0 i} \exp \left[-\frac{\left(\lambda-\lambda_{\sigma_{i}}\right)^{2}}{2 \Lambda_{\sigma_{i}}^{2}}\right], \quad(i=x, y, z) \\
& \delta_{j}(\lambda)=\delta_{0 j} \exp \left[-\frac{\left(\lambda-\lambda_{\delta_{j}}\right)^{2}}{2 \Lambda_{\delta_{j}}^{2}}\right], \quad(j=x, y, z)
\end{aligned}
$$

where the parameters $\lambda_{\sigma}\left(\lambda_{\delta}\right), \Lambda_{\sigma}\left(\Lambda_{\delta}\right)$ and $\sigma_{0}\left(\delta_{0}\right)$ are the central wavelength, the rms width, and the maximum value of the function at the central wavelength of the spectral distribution of the effective width (effective correlation length) of the scattering medium, respectively.

Similarly, with the help of the relationship between the wavelength and frequency, i.e., $\lambda=2 \pi c / \omega$, Eq. (2) can be rewritten as

$$
S^{(i)}(\lambda)=B \exp \left[-\frac{2 \pi^{2} c^{2}}{\Gamma_{0}}\left(\frac{1}{\lambda}-\frac{1}{\lambda_{0}}\right)\right]
$$

After that, we substitute from Eq. (7) into Eq. (6), then substitute the result into Eq. (5), through a series of calculations, and then one can obtain the cross-spectral density function of the scattered field as follows:

$$
\begin{aligned}
W^{(s)}\left(r \mathbf{s}_{1}, r \mathbf{s}_{2}, \mathbf{s}_{0}, \lambda\right)= & \frac{S^{(i)}(\lambda)}{r^{2}} \frac{1}{C_{0}} \sum_{m=1}^{M}\left(\begin{array}{c}
M \\
m
\end{array}\right)(-1)^{m-1} A \\
& \times \sigma_{x}(\lambda) \sigma_{y}(\lambda) \sigma_{z}(\lambda) \delta_{x}(\lambda) \delta_{y}(\lambda) \delta_{z}(\lambda)
\end{aligned}
$$

where

$$
\begin{aligned}
A & =\exp \left[-\frac{1}{2} k^{2}\left(s_{2 x}-s_{1 x}\right)^{2} \sigma_{x}^{2}(\lambda)\right] \exp \left[-\frac{1}{2} k^{2}\left(s_{2 y}-s_{1 y}\right)^{2} \sigma_{y}^{2}(\lambda)\right] \\
& \times \exp \left[-\frac{1}{2} k^{2}\left(s_{2 z}-s_{1 z}\right)^{2} \sigma_{z}^{2}(\lambda)\right] \exp \left[-\frac{1}{2} k^{2}\left(\frac{s_{1 x}+s_{2 x}}{2}-s_{0 x}\right)^{2} m \delta_{x}^{2}(\lambda)\right] \\
& \times \exp \left[-\frac{1}{2} k^{2}\left(\frac{s_{1 y}+s_{2 y}}{2}-s_{0 y}\right)^{2} m \delta_{y}^{2}(\lambda)\right] \exp \left[-\frac{1}{2} k^{2}\left(\frac{s_{1 z}+s_{2 z}}{2}-s_{0 z}\right)^{2} m \delta_{z}^{2}(\lambda)\right]
\end{aligned}
$$


If we let the two position vectors in the cross-spectral density function coincide, i.e., $\mathbf{s}_{1}=\mathbf{s}_{2}=\mathbf{s}$ the normalized spectral density of the scattered field can be obtained, with the form of

$$
\begin{aligned}
& S^{(s)}\left(r \mathbf{s}, \mathbf{s}_{0} ; \lambda\right)=\frac{s^{(i)}(\lambda)}{r^{2}} \frac{1}{C_{0}} \sum_{m=1}^{M}\left(\begin{array}{c}
M \\
m
\end{array}\right)(-1)^{m-1} \sigma_{x}(\lambda) \sigma_{y}(\lambda) \sigma_{z}(\lambda) \delta_{x}(\lambda) \delta_{y}(\lambda) \delta_{z}(\lambda) \\
& \quad \times \exp \left[-\frac{1}{2} k^{2}\left(s_{x}-s_{0 x}\right)^{2} m \delta_{x}^{2}(\lambda)\right] \exp \left[-\frac{1}{2} k^{2}\left(s_{y}-s_{0 y}\right)^{2} m \delta_{y}^{2}(\lambda)\right] \\
& \quad \times \exp \left[-\frac{1}{2} k^{2}\left(s_{z}-s_{0 z}\right)^{2} m \delta_{z}^{2}(\lambda)\right]
\end{aligned}
$$

In addition, the spectral degree of coherence of the far-zone scattered field also plays an important role on the description of the characteristics of the scattered light field, and the degree of coherence can be deduced from the cross-spectral density and the spectral density of the scattered field, by the following definition [29]:

$$
\mu^{(s)}\left(r \mathbf{s}_{1}, r \mathbf{s}_{2}, \mathbf{s}_{0}, \lambda\right)=\frac{W^{(s)}\left(r \mathbf{s}_{1}, r \mathbf{s}_{2}, \mathbf{s}_{0}, \lambda\right)}{\sqrt{S^{(s)}\left(r \mathbf{s}_{1}, \mathbf{s}_{0}, \lambda\right)} \sqrt{S^{(s)}\left(r \mathbf{s}_{2}, \mathbf{s}_{0}, \lambda\right)}}
$$

Upon substituting from Eqs. (13) and (15) into Eq. (16), we can obtain the spectral degree of coherence in the far-zone scattered field as follows:

$$
\begin{aligned}
\mu^{(s)}\left(r \mathbf{s}_{1}, r \mathbf{s}_{2}, \mathbf{s}_{0}, \lambda\right) & =\exp \left[-\frac{1}{2} k^{2}\left(s_{2 x}-s_{1 x}\right)^{2} \sigma_{x}^{2}(\lambda)\right] \exp \left[-\frac{1}{2} k^{2}\left(s_{2 y}-s_{1 y}\right)^{2} \sigma_{y}^{2}(\lambda)\right] \\
& \times \exp \left[-\frac{1}{2} k^{2}\left(s_{2 z}-s_{1 z}\right)^{2} \sigma_{z}^{2}(\lambda)\right]
\end{aligned}
$$

It is shown from the Eq. (15) and Eq. (17) that $\delta_{j}$ and $\sigma_{i}$ have a great influence on the normalized spectral density and the spectral degree of coherence, respectively.

\section{Numerical results}

In this section, we will present some numerical results to show the influence of the structural parameters of the scattering medium on the distribution of the far-zone scattered field. In Figs. 2-4, we will present the distributions of the normalized spectral density with different parameters. Figure 2 presents the effect of the rms width of the effective correlation length of the scattering medium on the distribution of the normalized spectral density. As shown in Fig. 2a-2 c, when we decrease the value of $\Lambda_{\delta x} / \Lambda_{\delta y}$ from $1 / 2$ to $1 / 4$, the angular width of $x$ direction of the distribution of the scattered nor- 


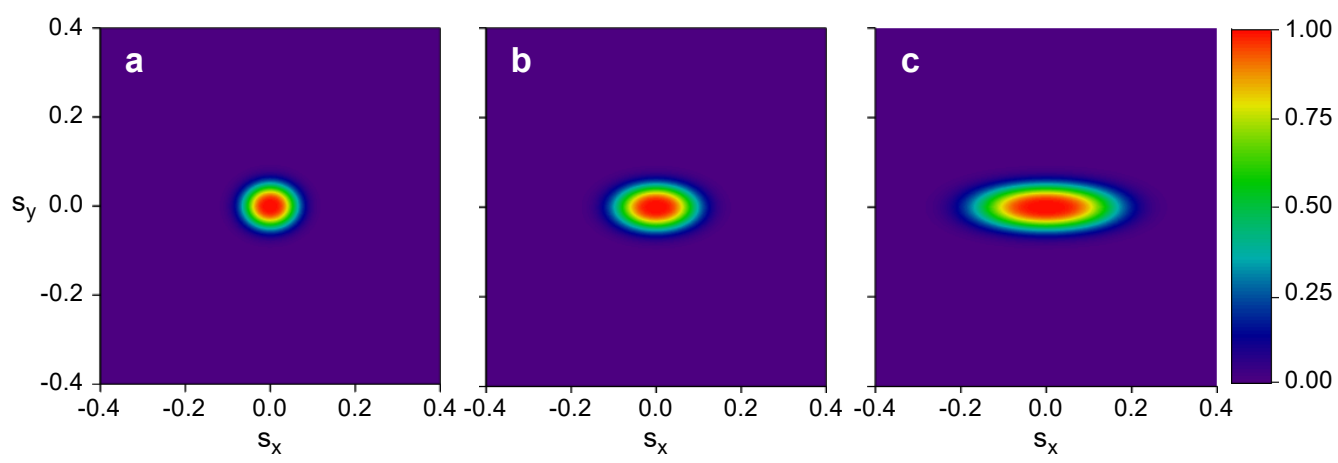

Fig. 2. Illustration of the normalized spectral density of the far-zone scattered field by Eq. (15). The parameters for calculations are: $M=2, \lambda=6.328 \times 10^{-7} \mathrm{~m}, \lambda_{\sigma x}=\lambda_{\sigma y}=\lambda_{\sigma z}=1.25 \times 10^{-6} \mathrm{~m}, \Lambda_{\sigma x}=\Lambda_{\sigma y}=$ $\Lambda_{\sigma z}=2.25 \lambda, \sigma_{0 x}=\sigma_{0 y}=\sigma_{0 z}=30 \lambda, \lambda_{\delta x}=\lambda_{\delta y}=\lambda_{\delta z}=0.25 \times 10^{-6} \mathrm{~m}, \delta_{0 x}=\delta_{0 y}=\delta_{0 z}=6 \lambda, \Lambda_{\delta y}=\Lambda_{\delta z}=1.5 \lambda$, (a) $\Lambda_{\delta x} / \Lambda_{\delta y}=1: 2$, (b) $\Lambda_{\delta x} / \Lambda_{\delta y}=1: 3$, and (c) $\Lambda_{\delta x} / \Lambda_{\delta y}=1: 4$.

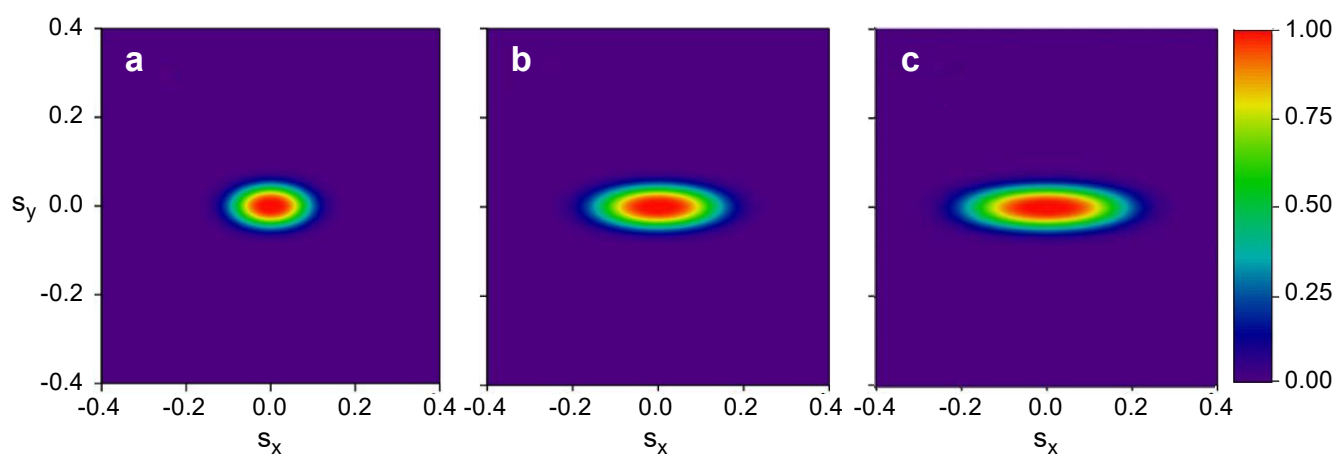

Fig. 3. Illustration of the normalized spectral density of the far-zone scattered field by Eq. (15). The parameters for calculations are: $M=2, \lambda=6.328 \times 10^{-7} \mathrm{~m}, \lambda_{\sigma x}=\lambda_{\sigma y}=\lambda_{\sigma z}=1.25 \times 10^{-6} \mathrm{~m}, \Lambda_{\sigma x}=\Lambda_{\sigma y}=$ $\Lambda_{\sigma z}=2.25 \lambda, \sigma_{0 x}=\sigma_{0 y}=\sigma_{0 z}=30 \lambda, \delta_{0 x}=\delta_{0 y}=\delta_{0 z}=6 \lambda, \Lambda_{\delta x}=\Lambda_{\delta y}=\Lambda_{\delta z}=0.45 \lambda, \lambda_{\delta y}=\lambda_{\delta z}=0.6 \times 10^{-6} \mathrm{~m}$, (a) $\lambda_{\delta x} / \lambda_{\delta y}=1: 2$, (b) $\lambda_{\delta x} / \lambda_{\delta y}=1: 3$, and (c) $\lambda_{\delta x} / \lambda_{\delta y}=1: 4$.

malized spectral density becomes larger. This phenomenon shows that the rms width of the effective correlation length plays a significant role on the distribution of the normalized spectral density of the far-zone scattered field.

Figure 3 shows the influence of the value of the center wavelength of the effective correlation length of the scattering medium on the normalized spectral density in the far-zone scattered field. It is shown that when the value of $\lambda_{\delta x} / \lambda_{\delta y}$ becomes smaller and smaller, the angular width of $x$ direction of the distribution of the normalized spectral density will be larger. This phenomenon shows that the center wavelength of the effective correlation length of the scattering medium also has a great effect on the distribution of the normalized spectral density of the far-zone scattered field.

In Fig. 4, we present the influence of the maximum value of the center wavelength of the effective correlation length of the scattering medium on the distribution of the 


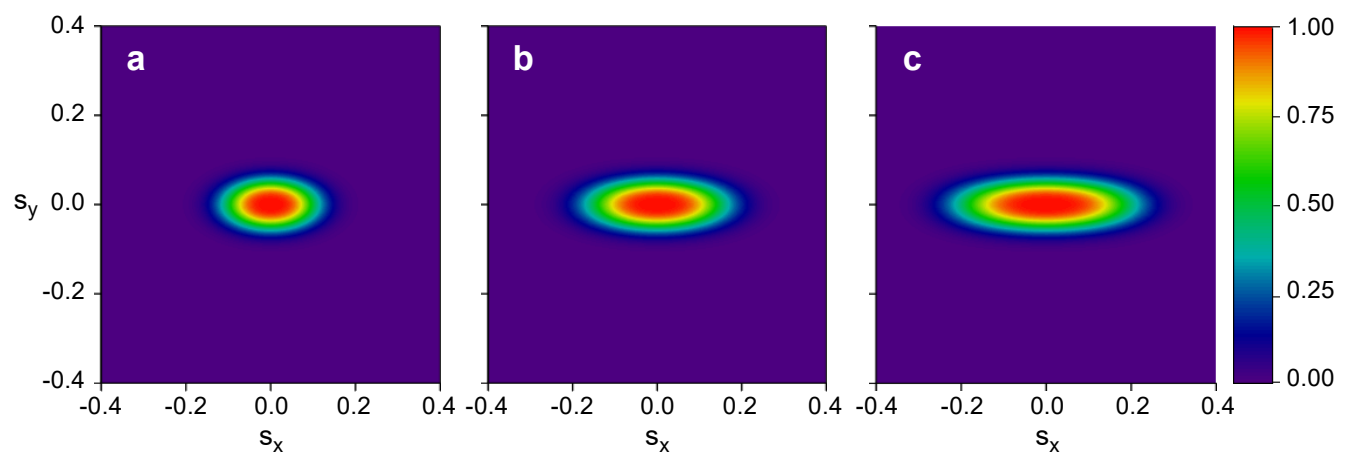

Fig. 4. Illustration of the normalized spectral density of the far-zone scattered field by Eq. (15). The parameters for calculations are: $M=2, \lambda=6.328 \times 10^{-7} \mathrm{~m}, \lambda_{\sigma x}=\lambda_{\sigma y}=\lambda_{\sigma z}=1.25 \times 10^{-6} \mathrm{~m}, \Lambda_{\sigma x}=\Lambda_{\sigma y}=$ $\Lambda_{\sigma z}=2.25 \lambda, \sigma_{0 x}=\sigma_{0 y}=\sigma_{0 z}=30 \lambda, \Lambda_{\delta x}=\Lambda_{\delta y}=\Lambda_{\delta z}=0.45 \lambda, \lambda_{\delta x}=\lambda_{\delta y}=\lambda_{\delta z}=0.25 \times 10^{-6} \mathrm{~m}, \delta_{0 y}=\delta_{0 z}=$ $=12 \lambda$, (a) $\delta_{0 x} / \delta_{0 y}=1: 2$, (b) $\delta_{0 x} / \delta_{0 y}=1: 3$, and (c) $\delta_{0 x} / \delta_{0 y}=1: 4$.

normalized spectral density in the far-zone scattered field. It is shown that as the value of $\delta_{0 x} / \delta_{0 y}$ is getting smaller, the angular width of $x$ direction of the distribution of the scattered normalized spectral density of the far-zone scattered field will be larger. This phenomenon shows that the maximum value of the center wavelength of the effective correlation length is also an important element which affects the distribution of the normalized spectral density of the far-zone scattered field.

In Figs. 5-7, we will present the distributions of the spectral degree of coherence with different parameters. For the simplicity of the following discussion, we set one variable in the spectral degree of coherence along the direction of the $z$-axis and change the other variable, i.e., $s_{1}=(0,0,1)$ and $s_{2}=\left(s_{2 x}, s_{2 y},\left(1-s_{2 x}^{2}-s_{2 y}^{2}\right)^{1 / 2}\right)$. In Fig. 5, we present the influence of the rms width of the effective width on the distribution of the spectral degree of coherence in the far-zone scattered field. We change the value of rms width of the effective width along $x$ direction and hold the values along $y$ and $z$ directions. When we decrease the value of $\Lambda_{\sigma x} / \Lambda_{\sigma y}$ from $1 / 2$ to $1 / 4$, one can find that
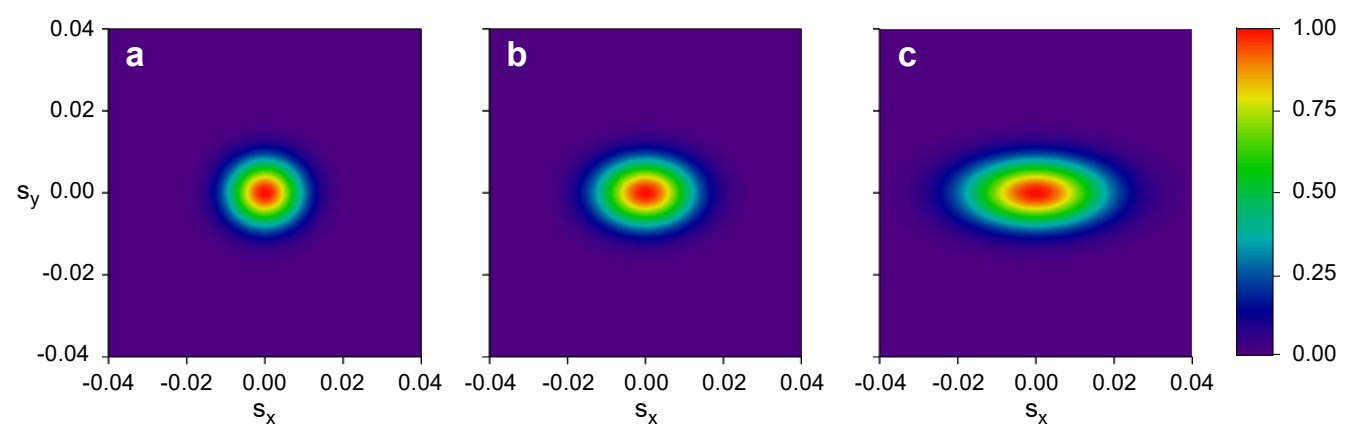

Fig. 5. Illustration of the spectral degree of coherence of the far-zone scattered field by Eq. (17). The parameters for calculations are as follows: $\lambda=6.328 \times 10^{-7} \mathrm{~m}, \lambda_{\sigma x}=\lambda_{\sigma y}=\lambda_{\sigma z}=1.25 \times 10^{-6} \mathrm{~m}, \sigma_{0 x}=\sigma_{0 y}=$ $=\sigma_{0 z}=30 \lambda, \Lambda_{\sigma y}=\Lambda_{\sigma z}=3 \lambda$. (a) $\Lambda_{\sigma x} / \Lambda_{\sigma y}=1: 2$, (b) $\Lambda_{\sigma x} / \Lambda_{\sigma y}=1: 3$, and (c) $\Lambda_{\sigma x} / \Lambda_{\sigma y}=1: 4$. 
the angular width of $x$ direction of the distribution of the degree of coherence becomes larger and larger. It is shown that the rms width of the effective width can affect the distribution of the degree of coherence.

In Fig. 6, the influence of the maximum value of the center wavelength of the effective width on the distribution of the spectral degree of coherence in the far-zone scattered field is discussed. From Figs. $6 \mathbf{a}-6 \mathbf{c}$, one can find that the smaller is the value of $\sigma_{0 x} / \sigma_{0 y}$ the larger will be the angular width of the degree of coherence distribution in $x$ direction. It is shown that the maximum value of the center wavelength of effective width also has a great influence on the distribution of the degree of coherence.

In Fig. 7, the influence of the center wavelength of the effective width on the distribution of the spectral degree of coherence in the far-zone scattered field is presented. One can clearly see from Figs. $7 \mathbf{a}-7 \mathbf{c}$ that the anisotropic distribution of the spectral degree of coherence becomes more obvious when we increase the value of $\lambda_{\sigma x} / \lambda_{\sigma y}$ from 2 to 4 . This phenomenon shows that the center wavelength of the effective width also has a great impact on the distribution of the degree of coherence.
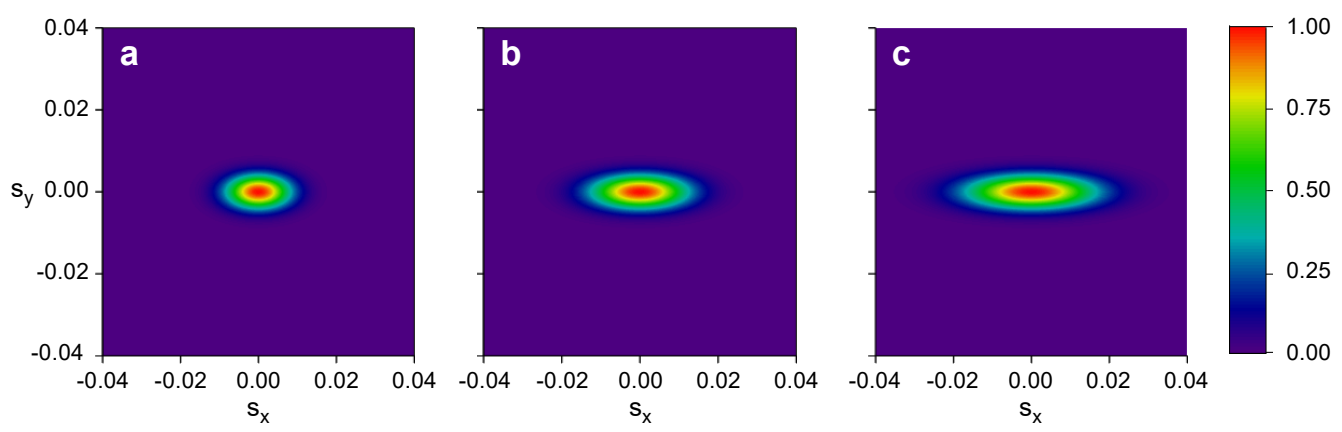

Fig. 6. Illustration of the spectral degree of coherence of the far-zone scattered field by Eq. (17). The parameters for calculations are as follows: $\lambda=6.328 \times 10^{-7} \mathrm{~m}, \Lambda_{\sigma x}=\Lambda_{\sigma y}=\Lambda_{\sigma z}=2.25 \lambda, \lambda_{\sigma x}=\lambda_{\sigma y}=\lambda_{\sigma z}=$ $=1.25 \times 10^{-6} \mathrm{~m}, \sigma_{0 y}=\sigma_{0 z}=60 \lambda$. (a) $\sigma_{0 x} / \sigma_{0 y}=1: 2$, (b) $\sigma_{0 x} / \sigma_{0 y}=1: 3$, and (c) $\sigma_{0 x} / \sigma_{0 y}=1: 4$.
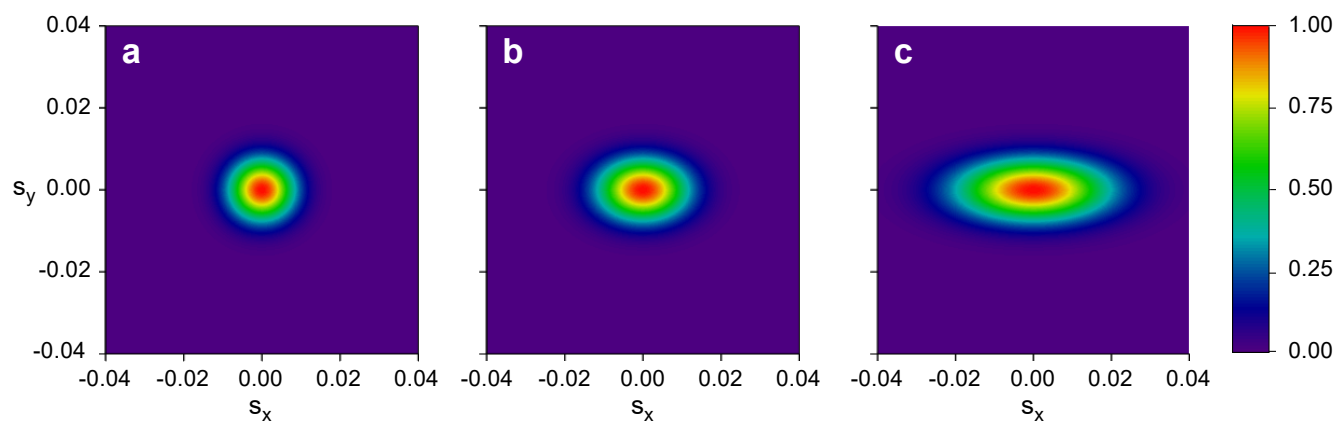

Fig. 7. Illustration of the spectral degree of coherence of the far-zone scattered field by Eq. (17). The parameters for calculations are as follows: $\lambda=6.328 \times 10^{-7} \mathrm{~m}, \Lambda_{\sigma x}=\Lambda_{\sigma y}=\Lambda_{\sigma z}=2.25 \lambda, \sigma_{0 x}=\sigma_{0 y}=\sigma_{0 z}=$ $=30 \lambda, \lambda_{\sigma y}=\lambda_{\sigma z}=0.65 \times 10^{-6} \mathrm{~m}$. (a) $\lambda_{\sigma x} / \lambda_{\sigma y}=2: 1$, (b) $\lambda_{\sigma x} / \lambda_{\sigma y}=3: 1$, and (c) $\lambda_{\sigma x} / \lambda_{\sigma y}=4: 1$. 


\section{Conclusion}

In summary, we discussed a scattering model of anisotropic semi-soft-boundary medium with spectral dependence. It is shown that the rms width, the center wavelength, and the maximum value of the center wavelength of the effective correlation length have a significant effect on the distribution of the normalized spectral density. In addition, the rms width, center wavelength, and maximum value of the center wavelength of the effective width also have a great influence on the distribution of the spectral degree of coherence of the far-zone scattered field. Owing to the discussion of this manuscript, we have further understood the properties of the anisotropic semi-soft boundary medium with spectral dependence, and these results may have potential application in the weak scattered field.

Acknowledgment - This research was supported by the National Natural Science Foundation of China (NSFC) under Grants 11404231, 61475105.

\section{References}

[1] Visser T.D., Fischer D.G., Wolf E., Scattering of light from quasi-homogeneous sources by quasi -homogeneous media, Journal of the Optical Society of America A 23(7), 2006, pp. 1631-1638, DOI: 10.1364/JOSAA.23.001631.

[2] Sahin S., Gbur G., Korotkova O., Scattering of light from particles with semisoft boundaries, Optics Letters 36(20), 2011, pp. 3957-3959, DOI: 10.1364/OL.36.003957.

[3] Wang T., Ji X., ZhaO D., Equivalence theorem for the spectral density of light waves on weak scattering, Optics Letters 39(13), 2014, pp. 3837-3840, DOI: 10.1364/OL.39.003837.

[4] Chang L., Li J., Reciprocal relations for third-order correlation between intensity fluctuations of light scattered from a quasi-homogeneous medium, IEEE Photonics Journal 9(2), 2017, article 6100507, DOI: 10.1109/JPHOT.2017.2683942.

[5] Carter W.H., Wolf E., Scattering from quasi-homogeneous media, Optics Communications 67(2), 1988, pp. 85-90, DOI: 10.1016/0030-4018(88)90309-4.

[6] XIN Y., HE Y., CHEN Y., Li J., Correlation between intensity fluctuations of light scattered from a quasi-homogeneous random media, Optics Letters 35(23), 2010, pp. 4000-4002, DOI: 10.1364/ OL.35.004000.

[7] Li J., Chang L., Spectral shifts and spectral switches of light generated by scattering of arbitrary coherent waves from a quasi-homogeneous media, Optics Express 23(13), 2015, pp. 16602-16616, DOI: $10.1364 / \mathrm{OE} .23 .016602$.

[8] XiN Y., CHen Y., Zhao Q., ZHou M., Beam radiated from quasi-homogeneous uniformly polarized electromagnetic source scattering on quasi-homogeneous media, Optics Communications 278(2), 2007, pp. 247-252, DOI: 10.1016/j.optcom.2007.06.030.

[9] Li J., Qin Y., Zhou S., Fourth-order correlation statistics of an electromagnetic plane wave scattered by a quasi-homogeneous medium, Journal of Optics 13(11), 2011, article 115702, DOI: 10.1088/ 2040-8978/13/11/115702.

[10] Korotкova O., Can a sphere scatter light producing rectangular intensity patterns?, Optics Letters 40(8), 2015, pp. 1709-1712, DOI: 10.1364/OL.40.001709.

[11] Fischer D.G., Wolf E., Inverse problems with quasi-homogeneous random media, Journal of the Optical Society of America A 11(3), 1994, pp. 1128-1135, DOI: 10.1364/JOSAA.11.001128. 
[12] Baleine E., Dogariu A., Variable-coherence tomography for inverse scattering problems, Journal of the Optical Society of America A 21(10), 2004, pp. 1917-1923, DOI: 10.1364/JOSAA.21.001917.

[13] WANG T., Zhao D., Determination of correlation function of scattering potentials of a random medium from the scattered spectral density, Physics Letters A 375(3), 2011, pp. 780-783, DOI: 10.1016/ j.physleta.2010.11.049.

[14] LI J., Determination of correlation function of scattering potential of random medium by Gauss vortex beam, Optics Communications 308, 2013, pp. 164-168, DOI: 10.1016/j.optcom.2013.06.059.

[15] GBur G., Wolf E., Determination of density correlation functions from scattering of polychromatic light, Optics Communications 168(1-4), 1999, pp. 39-45, DOI: 10.1016/S0030-4018(99)00214-X.

[16] Lahiri M., Wolf E., Fischer D.G., Shirai T., Determination of correlation functions of scattering potentials of stochastic media from scattering experiments, Physical Review Letters 102(12), 2009, article 123901, DOI: 10.1103/PhysRevLett.102.123901.

[17] Tong Z., Konotкova O., Theory of weak scattering of stochastic electromagnetic fields from deterministic and random media, Physical Review A 82(3), 2010, article 033836, DOI: 10.1103/ PhysRevA.82.033836.

[18] Zноu J., Zнао D., Scattering of a random electromagnetic beam from a random medium with spectral dependence, Optics Express 25(15), 2017, pp. 17114-17127, DOI: 10.1364/OE.25.017114.

[19] Zhang Y., ZhaO D., Hanbury Brown-Twiss effect with partially coherent electromagnetic beams scattered by a random medium, Optics Communications 350, 2015, pp. 1-5, DOI: 10.1016/j.optcom. 2015.03.075.

[20] Ding Y., Zhaо D., Random medium model for producing optical coherence lattice, Optics Express 25(21), 2017, pp. 25222-25233, DOI: 10.1364/OE.25.025222.

[21] Wang X., Liu Z., Huang K., Zhu D., Spectral changes of cosine-Gaussian-correlated Schell-model beams with rectangular symmetry scattered on a deterministic medium, Journal of the Optical Society of America A 33(10), 2016, pp. 1955-1960, DOI: 10.1364/JOSAA.33.001955.

[22] Wang T., Zhao D., Determination of pair-structure factor of scattering potential of a collection of particles, Optics Letters 35(3), 2010, pp. 318-320, DOI: 10.1364/OL.35.000318.

[23] Sahin S., Korotкova O., Scattering of scalar light fields from collections of particles, Physical Review A 78(6), 2008, article 063815, DOI: 10.1103/PhysRevA.78.063815.

[24] Dogariu A., Wolf E., Spectral changes produced by static scattering on a system of particles, Optics Letters 23(17), 1998, pp. 1340-1342, DOI: 10.1364/OL.23.001340.

[25] Mei Z., Korotкova O., Random light scattering by collections of ellipsoids, Optics Express 20(28), 2012, pp. 29296-29307, DOI: 10.1364/OE.20.029296.

[26] Zhaо D., Wang T., Direct and inverse problems in the theory of light scattering, [In] Progress in Optics, [Ed.] E. Wolf, Vol. 57, 2012, pp. 261-308, DOI: 10.1016/B978-0-44-459422-8.00005-9.

[27] Shchepakina E., Korotкova O., Spectral Gaussian Schell-model beams, Optics Letters 38(13), 2013, pp. 2233-2236, DOI: 10.1364/OL.38.002233.

[28] JiAng Z., HuAng Z., Cheng K., Wang T., Spectral behavior of light waves on scattering from a semi -soft-boundary medium with spectral dependence, Journal of the Optical Society of America A 36(3), 2019, pp. 443-449, DOI: 10.1364/JOSAA.36.000443.

[29] Wolf E., Introduction to the Theory of Coherence and Polarization of Light, Cambridge University Press, Cambridge, 2007.

[30] JIANG Z., ZHU L., Ji X., CHENG K., WANG T., Far-zone behaviors of light waves on scattering from anisotropic quasi-homogeneous media with semisoft boundaries, Journal of the Optical Society of America A 34(8), 2017, pp. 1463-1468, DOI: 10.1364/JOSAA.34.001463.

Received May 4, 2020

in revised form June 22, 2020 